\title{
TENDENCIA DA SUB-NOTIFICAÇÃO DE CASOS NO DECORRER DA EPIDEMIA DE MENINGITE MENINGOCÓCICA OCORRIDA NO ESTADO DE SÃO PAULO, BRASIL, NO PERÍODO DE $1971 / 75$
}

Gilberto Ribeiro Arantes:

Antonio Ruffino-Netto:*

ARAntes, G. R. \& Ruffino-Netto, A. Tendência da sub-notificąão de casos no decorrer da epidemia de meningite meningocócica ocorrida no Estado de São Paulo, Brasil, no periodo de 1971/75. Rev. Saúde públ., S. Palto, 11: 182-7, 1977.

Resumo: A sub-notificaçäo de casos de meningite no decorrer de uma epidemia de meningite meningocócica foi estudada em uma região do Estado de $S$. Paulo, Brasil. Os casos conhecidos através dos atestados de óbito (não notificados em vida) foram usados como medida indireta da sub-notificação. Esta, que chegou a 37,6\% em 1971, caill para 2,1\% em 1975. Foi possivel quantificá-la e demonstrar que seguiu uma funçāo exponencial negativa com o tempo. A aplicaçāo da metodologia apresentada, em amostra casilal e probabilistica, proporcionará um melhor conhecimento da incidência da doença, mais adequado ao estudo da tendência da epidemia, o que permitirá estimar com maior segurança o impacto epidemiológico provocado pelas medidas adotadas para controle da mesma.

Untrermos: Meningite meningocócica, Süo Paulo, Brasil, Epidemia, sub-notificação.

Apenas uma parcela dos casos incidentes de uma moléstia de notificação compulsória é notificada às autoridades sanitárias; da parcela não notificada uma parte acaba sendo conhecida pelos órgãos oficiais através dos atestados de óbito, porém os casos não notificados que sobrevivem à moléstia jamais são computados, por isso perdidos para estimativas de morhidade.
É possivel que no decorrer de epidemia prolongada, seja pela melhor atuação das autoridades sanitárias junto aos médicos e hospitais, ou devido à grande ênfase concedida ao assunto pelos órgãos da imprensa escrita e falada, a sub-notificação seja menor. Aproveitando a recente epidemia de meningite meningocócica que se iniciou no Estado de São Paulo em 1971, propusemo-

* Da Divisão de Estudos e Programas da Coordenadoria de Suude da Comunidade, da Secretarla da Saúde do Estado de São Paulo - Avenida S. Luiz, 99 - Săo Paulo, SP - Brasil.

** Do Departamento de Medicina Social da Faculdade de Medicina de Ribeirão Preto da Uini. versidade de São Paulo - Riheirão Preto, SP $\ldots$ Brisil. 
ARANTES, G. R. \& RUFFINO-NETTO, A. - Tendência da sub-notificação de casos no decorier da epidemia de meningite meningocócica ocorrida no Estado de São Paulo, Brasil, no períno de 1971/75. Rer. Saúde públ., S. Pauln 11:182-7, 1977

nos verificar se realmente diminuiu a subnotificação de casos de meningite, bem como tentar medir a intensidade desse declínio.

\section{MATERIAL E METODOS}

Foram usados como parâmetros de incidência notificada e não notificada os casos autóctones de meningite de qualquer etiologia, notificados aos Centros de Saúde, e os casos não notificados em vida, porém conhecidos através do atestado de óbito. A área estudada foi a 6a Regional de Saude de São Paulo ${ }^{4}$ que abrange 80 municipios na região nordeste do Estado, cuja sede administrativa está situada na ciclade de Ribeirão Preto.

Até 1971, us boletins epidemiológicos consolidados em nivel regional continham os casos notificados e os "casos" conhecidos através dos respectivos óbitos. Nos anos de 1972 a 1974, novos boletins foram instituidos: contendo apenas os casos notificados sem os nomes dos pacientes; devido a essa omissão não era possivel verificar no mapa demógrafo-sanitário MDS * yuais os óbitos por meningite que não haviam sido notificados em vida. A partir de 1975 , esses boletins, embora nāo incluindo os "casos" conhecidos pelo óbito, voltaram a incluir os nomes dos pacientes ${ }^{3}$, permitindo assim aquela verificação no MDS.

Com a intenção de conhecer a proporção desses "casos" no total de casos conhecidos nos anos de 1972, 1973 e 1974, foi feito um levantamento "in loco" na fonte primária das informaçōes em 5 Centros de Saúde localizados em sedes de Distritos Sanitários ${ }^{1}$ (sub-regiões administrativas) a saber: Ribeirão Preto, Franca, Bebedouro, Jaboticabal e Araraquara. A fonte citada é c) livro de registro de casos notificados e chitos por moléstias contagiosas, conhecido antes da Reforma Administrativa da Secretaria da Saude como "B-12" e atualmente como "E-2". Para proceder ao levantamento das informaçōes preliminarmente arrolavam-se, mês a mês para todos os anos do periodo 1971-1975, em ordem alfabética, os obitos por meningite de pessoas residentes no município; a seguir procedia-se à leitura no E-2 do nome de cada caso notificado e confirmado para verificar se o mesmo constava da lista de óbitos com a finalidade de eliminá-lo desta se tivesse sido notificado em vida. Os remanescentes eram arrolados como "casos" conhecidos através do óbito e somados aos primeiros para ohter a incidência total.

\section{RESULTADOS E DISCUSSÃO}

Os resultados obtidos são apresentados na Tabela 1, onde o número de casos conhecidos através do atestado de óbito nos 5 municipios (que é una estimativa indireta de sub-notificaçāo ${ }^{5}$ ), nota-se que sua percentagem variou de $37,6 \%$ (em 1971) a $2,1 \%$ (em 1975) em relação ao total de casos conhecidos. As percentagens dos casos conhecidos atrayés do atestado de óbito são apresentados na Figura 1.

A observação desse gráfico mostra que as percentagens projetadas nas urdenadas parecem seguir uma função exponencial negativa em relação an tempo (projetado nas abcissas)

Sendo:

$y=$ percentual de casos de meningite conhecidos através do atestado de óbito;

$\mathrm{x}=$ tempo calendáriu.

Fazendo-se equivaler:

$\mathrm{x}=0$ para 0 ano de $1971 ; \mathrm{x}=1, \mathrm{x}=2$,

$\mathrm{x}=3$, e $\mathrm{x}=4$, para os anos respectivamente de 1972, 1973, 1974 e 1975,

* Relação nominal dos óbitos ocorridos no mês e respectivas causas, que os cartórios de paz enriam ao centro de Saúde da sua área de jurisdição 


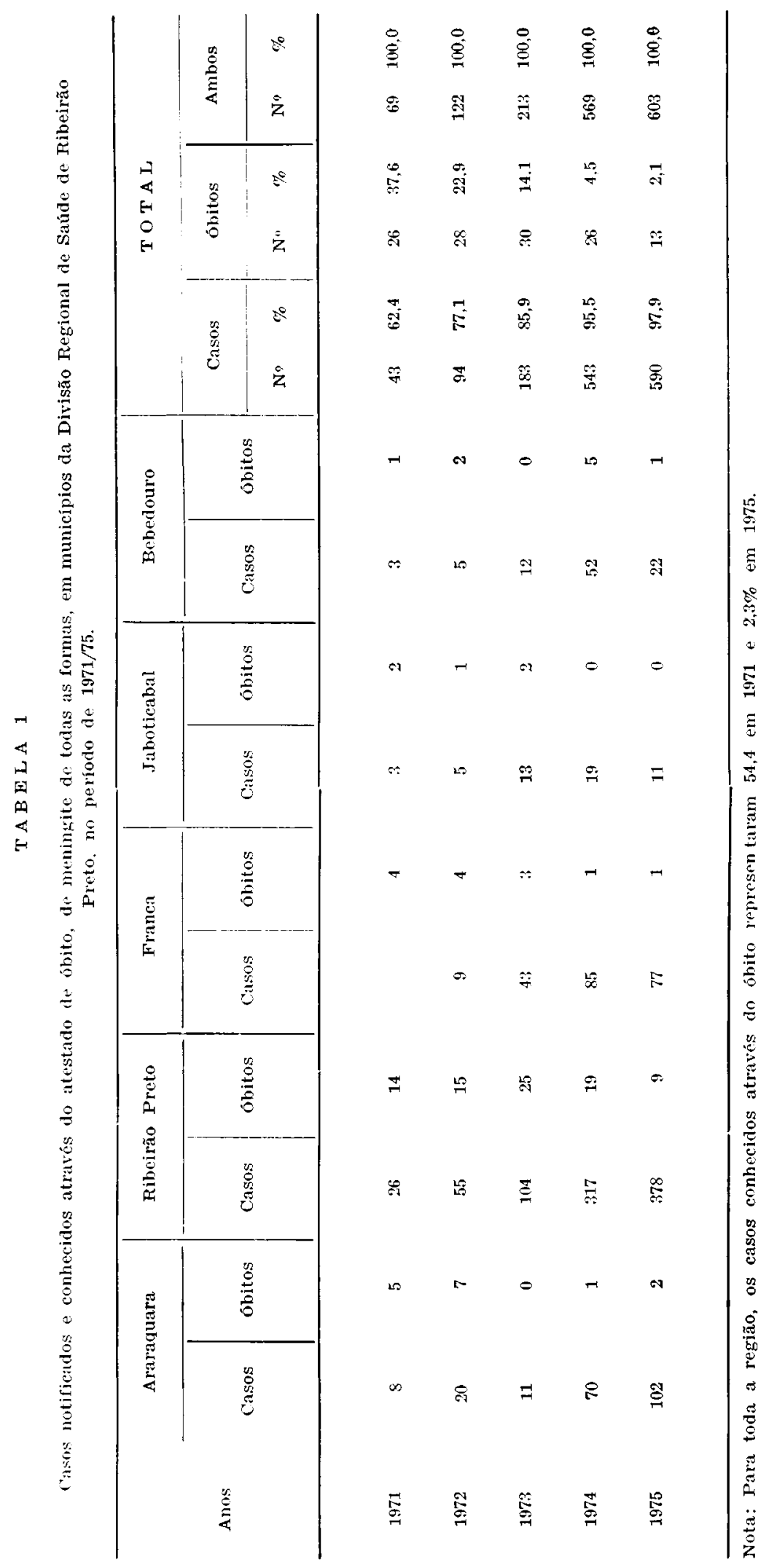


ARANTES, G. R. \& RUFFINO-NETTO, A. - Tendencia da sub-notificação de casos no decorrer da epidemia de meningite meningocócica ocorrida no Estado de São Paulo, Brasil, no período de 1971/75. Rev. Saude pübl., S. Paulo, 11:182-7, 1977.

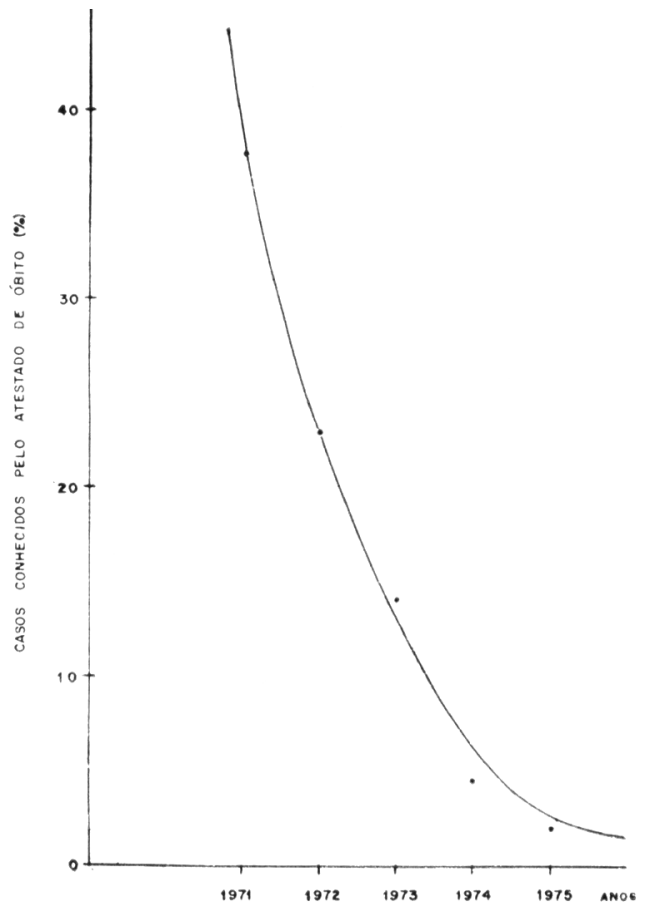

Fig. 1 - Percentagens de "casos" de meningite (todas as formas) conhecidos através dos 6 bitos. em 5 municípios da Divisão Regional de Saúde de Ribeirão Preto, de 1971 a 1975.

e projetando-se os valores de $x$ e $y$ em un gráfico en escala semi-logaritmica (Fig. 2) observa-se que a reta obtida corrobora a hipótese anterior, isto é, parece haver uma função do tipo $\log \mathrm{y}=\mathrm{a}-\mathrm{bx}$ ou

$$
y=y_{0} \quad 10^{-b x}
$$

Efetuando o ajustamento da função pelo método dos mínimos quadrados 1 obteve-se a equação:

$$
\begin{aligned}
\log y & =1,65-0,32 x, \\
\text { ou seja, } y & =44,67 \cdot 10^{-0,32 x}
\end{aligned}
$$

cujo teste de ajustamento de função 1 ("goodness of fit") pelo método dos $x^{2}$ resultou $\chi^{2}=2,748$, mostrando que não se poderia rejeitar a hipótese da função exponencial anteriormente aventada.

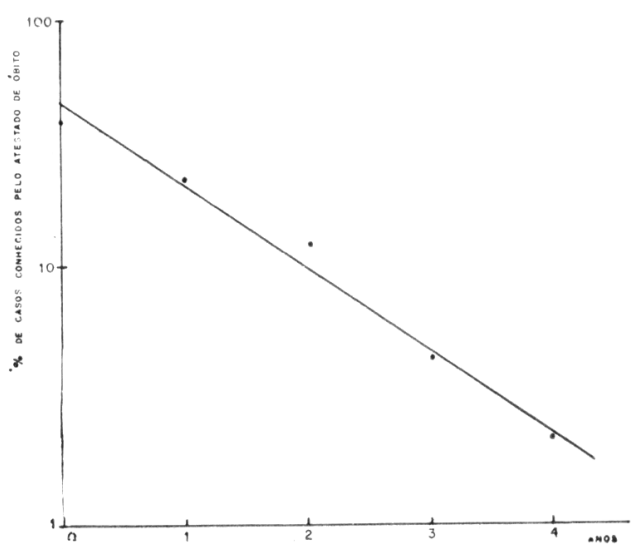

Fig. 2 - Percentagens de "casos" de meningite (todas as formas) conhecidos através dos óbitos. em 5 municípios da Divisão Regional de Saúde de Ribeirão Preto, de 1971 a 1975 (escalit semi-logaritmica).

Sendo y (que é em uma medida indireta da sub-notificação) uma função que cai exponencialmente com o tempo é de se supor que a "força de notificação" (que chamaremos de variável $z$ ) deva ter variado no período considerado através da relação $\mathrm{z}=(100-\mathrm{y}) \%$.

Conhecendo-se os dados referentes ao conjunto da DRS-6, nos anos de 1971 e 1975 , quando os casos conhecidos pelo atestado de óbito representaram respectivamente $54,4 \% \quad\left(y_{0}=54,4\right)$ de 112 casos e $2,3 \%$ de 1267 casos, e supondo-se que a velocidade de declinio da sub-notificação $\left(10^{-0,32 x}\right)$ encontrada nos 5 municipios fosse aplicável aos 80 municípios da região, a incidência total nos anos intermediários (1972, 1973 e 1974) seria aquela apresentada na Tabela 2. Acreditamos, contudo, que esta última suposição (qual seja, velocidade de declínio da sub-notificação $=10^{-0,32 x}$ para toda a DRS-6) não tenha suporte, pelas razões:

1. Os 5 municípios estudados não constituem uma amostra representativa (casual e probabilistica) da DRS-6. 
ARANTES, G. R. \& RUFFINO-NETTO, A. - Tendência da sub-notificação de casos no decorrer da epidemia de meningite meningocócica ocorrida no Estado de São Paulo, Brasil, no periodo de 1971/75. Rev. Saúde públ., S. Paulo, 11:182-7, 1977.

2. A conıparação das "sub-notificações" observadas nos 5 municipios (Tabela 1) mostra que as velucidades não foran as mesmas para todos.
3. A média inicial obtida nesses 5 municipios $(37,6 \%)$ foi muito diferente da sub-notificação média inicial de toda a região $(54,4 \%)$.

T A B E L A 2

Estimativa de casos notificados e casos conhecidos através do atestado de óbito, de meningite de todas as formas, na Divisão Regional de Saúde de Ribeirão Preto, 1971 a 1975.

\begin{tabular}{|c|c|c|c|c|c|}
\hline \multirow{2}{*}{ Anos } & \multirow{2}{*}{$\begin{array}{c}\text { Casos } \\
\text { notifi- } \\
\text { cados }\end{array}$} & \multicolumn{2}{|c|}{$\begin{array}{c}\text { Casos conhecidos pelo } \\
\text { atestado de óbito }\end{array}$} & \multicolumn{2}{|c|}{ Total de casos } \\
\hline & & $\%$ & $N o$ & No & $\%$ \\
\hline 1971 & 51 & $54,4 \%$ & 61 & 112 & $100 \%$ \\
\hline 1972 & 3338 & $26.0 \%$ & 119 & 457 & $100 \%$ \\
\hline 1973 & 540 & $12,4 \% *$ & 77 & 617 & $100 \%$ \\
\hline 1974 & 1305 & $5.9 \% *$ & $8: 3$ & 1388 & $100 \%$ \\
\hline 1975 & 1237 & $2,3 \%$ & 301 & 1267 & $100 \%$ \\
\hline
\end{tabular}

* Calculando a partir de $y=54,4,10^{-0,32 x}$

É possivel que os fatores que influenciaram as comunidades, no sentido de não serem omitidas as notificações da doença em pauta tenham atuado com intensidade diferente em cada comunidade, em decorrência de caracteristicas culturais de cada uma. É possivel também que as diferenças iniciais tenham corrido por conta de em uma maior sub-notificação nas pequenas comunidades, que foi se atenuando na medida em que a atuação do órgão Regional de Saúde foi se intensificando.

Por estas razóes, o "fator de correção" obtido não pode ainda ser usado para corrigir o conjunto dos dados regionais, mês a mês, nos anos estudados.

Julgamos que apenas uma amustra representativa dos municípios permitiria elucidar 0 tipo de função $Y$ em relação à $X$ para toda a DRS-b. É válida contudo a metodologia apresentada para aplicação em outras regiões.

Sugerimos que as sub-notificaçoes nos anos de 1972. 1973 e 1974 sejam pesquisadas e quantificadas para que se possam construir curvas de incidencia da doença mais adequadas a uma análise, interpretação e avaliação do impacto provocado pelas medidas adotadas para o controle da epidemia.

\section{CONCLUSAO}

Foi possivel quantificar a "sub-notificação" dos casos de meningite em 5 münicípios da DRS-6, que em termos percentuais seguiu uma função exponencial negativa com o tempo, no período de 1971 a 1975. 
ARANTES. G. R. \& RUFFINO-NETTO. A. - Tendéncia da sub-notificação de casos no decorrer da epidemia de meningite meningocócica ocorrida no Estado de São Paulo, Brasil, no periodo de 1971/75. Rer. Saúde públ., S. Paulo, 11:182-7, 1977

ARAnTES, G. R. \& Ruffino-NetTo, A. [Trends of under-reporting of cases during an outbreak of meningococcal meningitis in the State of $S$. Paulo (Brazil), from 1971 through 1975]. Rev. Saúde públ., S. Paulo, $11: 182-7,1977$.

ABSTRACT: A study of under-reporting of meningitis in five communities in the State of S. Paulo (Brazil), during an outbreak of meningococcal meningitis was undertaken. The number of "cases" (not notified during life) achieved through the examination of death records was used as an indirect measure of under-reporting. There was a variation in under-reporting during the epidemic period: it decreased from $37.6 \%$ in 1971 to $2.1 \%$ in 1975 , following a negative exponential pathway, at a measurable rate. The application of the methodology presented in this paper would provide a more accurate knowledge of the incidence of the disease allowing a better interpretation of the trends of the epilemic in order to evaluate the effectiveness of policies adopted to control it.

Uniterms: Meningococcal meningitis, S. Paulo, Brazil. Epidemic under-reporting.

\section{REF'ERENCIAS BIBLIOGRAFICAS}

1. LINDGREN. B. W. \& McELRATH. G. W. Introduction to probability and statistics. 2nd ed. New York, Collier-Macmillan Student Editions, 1976.

2. SĀO PAUlo (Estado). Secretaria da Saúde. Norma técnica. SS-7/71. Diário Oficial do Estado, $12 \mathrm{fev} 1971$. p. $24-30$.

3. SĀO PAULO (Estado). Secretaria da Saúde. Norma técnica: $\mathrm{SS} / 71$ refor. mulada. Diario Oficial do Estado, 23 fer. 1974, p. 41-2.

4. São PAULO (Estado). Secretaria da Saúde. Reforma administrativa: coletanea de textos legais e regulamentares. São Paulo. 1971.

5. SWAROOP, S. Introduction to health statistics. Edinburgh Livingstone, 1960.

Recebido para publicaşão em 29/10/19\%6. Aprovado para publicasão em 17/12/1976. 